\title{
Light Charged Higgs Searches in the Hadronic Mode with ATLAS
}

\section{Elias Coniavitis*}

on behalf of the ATLAS Collaboration

Uppsala University

E-mail: elias.coniavitisecern.ch

\begin{abstract}
The channel in which a $H^{+}$is produced in $t \bar{t}$ events with both the $\tau$ (from the $H^{+}$) and the $W$ boson decaying hadronically has a relatively high cross section, making it a priori one of the most promising in searches for a $H^{+}$lighter than the top quark. On the other hand the absence of leptons as well as the high hadronic activity in the event is a challenge, in particular for triggering purposes. The ATLAS simulation study for this channel is presented along with its expected discovery reach.
\end{abstract}

Prospects for Charged Higgs Discovery at Colliders

16-19 September 2008

Uppsala, Sweden

\footnotetext{
* Speaker.
} 


\section{Introduction}

Charged Higgs bosons are predicted by a large number of models containing a non-minimal Higgs sector, such as the Higgs sector of the Minimal Supersymmetric extension of the Standard Model (MSSM) or Two Higgs Doublet Models in general. Thus the discovery of a charged Higgs boson would be an unambiguous sign of physics beyond the Standard Model. At tree level the MSSM Higgs sector is fully described by the $H^{+}$mass and the ratio of the two Higgs doublets' vacuum expectation values, $\tan \beta$. If the $H^{+}$has a mass below the top quark mass ("light" $H^{+}$), it is predominantly produced in top quark events, and decays to a $\tau$ lepton and its neutrino with a branching ratio very close to 1 , except for very small values of $\tan \beta$.

ATLAS is an all-purpose detector at the Large Hadron Collider (LHC) at CERN in Geneva. Detailed studies [1] have been performed to determine the potential of ATLAS for charged Higgs boson searches, using a number of possible production and decay modes [2]. In the study presented here, the hadronic mode of a light $H^{+}$has been considered: the signal consists of $t \bar{t}$ events, in which one top quark decays to a bottom quark and a charged Higgs boson, which in turn decays to a hadronically decaying tau and its neutrino; while the other top quark decays to a bottom quark and a $W$ which decays to two jets. Several background processes have been considered: $t \bar{t}$ (all possible decay modes), single top, $W+$ jets and QCD dijets (in the $p_{\mathrm{T}}$ range of 140 to $1120 \mathrm{GeV}-$ events with a lower $p_{\mathrm{T}}$ are not expected to satisfy the event selection requiring several hard objects, while the cross section for events with higher $p_{\mathrm{T}}$ is relatively small). For further information on event simulation and cross section calculation, see [1].

\section{Trigger}

A trigger signature requiring a tau and missing energy (trigger $A$ ) or a tau and missing energy and three jets (trigger $B$ ) is used for this study (see [3] for more details on the ATLAS charged Higgs trigger). The trigger is one of the greatest challenges for this channel, since only about 9-17\% of the signal fires this trigger, depending on the mass point.

\section{Analysis}

The analysis for this channel consists of two sets of selection cuts followed by a likelihood discriminant. The first set of cuts is aimed at selecting events agreeing with the signal final state, while the second set of cuts is specifically aimed at reducing the QCD background by identifying the $t \bar{t}$ topology. Finally, the purpose of the likelihood is to discriminate between the signal and the SM-like $t \bar{t}$ background. Following a cut on the likelihood, the $H^{+}$transverse mass is reconstructed and the significance of the signal hypothesis is calculated using a shape-based profile likelihood method. The reconstruction of many high level physics objects, such as b- or $\tau$-tagged jets, is needed in charged Higgs boson searches. Dedicated ATLAS algorithms have been used for their reconstruction, and details about these can be found in [1]. 


\begin{tabular}{ll} 
Cuts I & Cuts II \\
\hline 1. exactly $1 \tau$-tagged jet with $p_{T}>35 \mathrm{GeV}$ & 6. $\left|m_{W}^{\text {rec }}-m_{W}\right|<30 \mathrm{GeV}$ \\
2. exactly $2 b$-tagged jets with $p_{T}>15 \mathrm{GeV}$ & 7. $\left|m_{t}^{\text {rec }}-m_{t}\right|<40 \mathrm{GeV}$ \\
3. at least 2 non-tagged jets with $p_{T}>15 \mathrm{GeV}$ & 8. $\Delta \phi\left(p_{T}^{\text {hardest top }}, p_{T}^{\text {softest top }}\right)>2.5$ \\
4. veto on isolated leptons with $p_{T}>5 \mathrm{GeV}$ & 9. $p_{T}^{\text {hardest top }} / p_{T}^{\text {softest top }}<2$ \\
5. $E_{T}^{\text {miss }}>40 \mathrm{GeV}$ (50 if event was fired by & \\
trigger $A$ only) &
\end{tabular}

Table 1: Applied selection cuts. A $b$-tagging cut is applied, yielding a $b$ jet reconstruction efficiency of about $70 \%$. For $\tau$ jets, a cut on the $\tau$-tagging variable is applied such that a reconstruction efficiency of about $32 \%$ is obtained for a pseudorapidity $|\eta|<1.5$, and a tighter cut (due to the high QCD jet fake-rate for large $\eta$ ) for $|\eta|>1.5$ leads to an efficiency of about $20 \%$ in this region.

\subsection{Selection Cuts}

Selection Cuts I The first set of cuts relates to the multiplicity and transverse momentum of the objects in the event, aiming to pick out the events resembling the signal final state. These cuts are detailed in the first column of Table 1.

$W$ and top quark Reconstruction After the first set of cuts, the $W$ is reconstructed from the pair of non $b$-tagged jets with an invariant mass closest to the $W$ mass. Its parent top quark is then reconstructed by pairing the reconstructed $W$ with the $b$ quark giving the invariant mass closest to the top quark mass. Due to the neutrinos in its decay products, the top quark that decayed to the charged Higgs boson cannot be fully reconstructed. However, it is still possible to extract information on its azimuthal angle and transverse momentum by adding the transverse momenta of the remaining $b$-tagged jet and the $\tau$ jet with the missing transverse momentum.

Selection Cuts II The second set of selection cuts aims to identify the topology of $t \bar{t}$ events, in order to discriminate against $\mathrm{QCD}$, as well as single top and $\mathrm{W}+\mathrm{jets}$ backgrounds, a relatively large amount of which still remain at this point. These cuts can be found in the second column of Table 1 .

\subsection{Likelihood}

At this point of the analysis, the majority of the remaining background consists of $t \bar{t}$ events where one $W$ decays hadronically and the other to a $\tau$ and a neutrino. In order to discriminate between the signal and this background a 2-class likelihood is used with $t \bar{t}$ (with at least one $e, \mu$ or $\tau$ ) as the only background class. The variables entering the likelihood are presented in Table 2 . They have been selected to reflect the heavier mass of the $\mathrm{H}^{+}$compared to the $W$, with its effects on the kinematics of the event; and the difference in $\tau$ polarization depending on the parent. This difference in polarization causes the leading track within the $\tau$ jet to be harder in average [ $₫$ and is exploited in variable I. Also, selected combinations of variables have been included, as correlations are not included in the definition of the likelihood used. The likelihood distribution obtained can be seen in Figure 1/(a). For an event to be kept, the obtained likelihood is required to have a value greater than 0.6 ( 0.8 for $m_{H^{+}}=150 \mathrm{GeV}$, due to the better signal-background separation). 


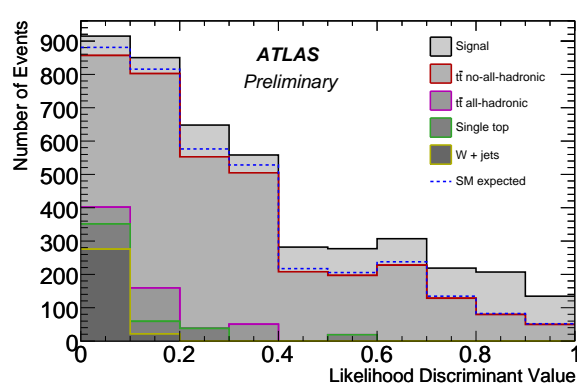

(a)

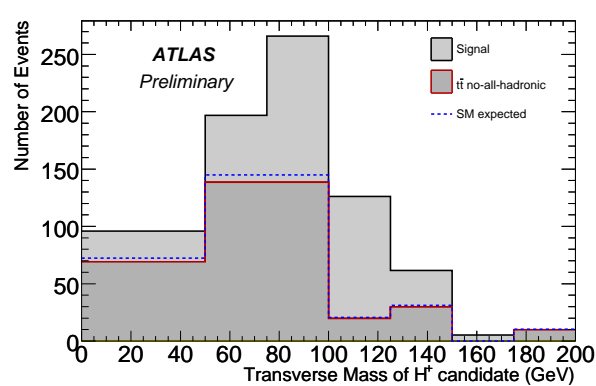

(b)

Figure 1: Likelihood distribution (a) and transverse mass (b) for an $\mathrm{H}^{+}$mass of $130 \mathrm{GeV}$ and the corresponding background, stacked and normalized to the cross section at $\tan \beta=20$. The blue dashed line shows what is expected from a Standard Model-only scenario.

$$
\begin{array}{llll}
\text { I. } & p_{T}^{l t} / p_{T}^{\tau} & \text { V. } & p_{T}^{\tau} / p_{T}^{b_{H^{+}}} \\
\text {II. } & 1-\cos \left(\Delta \phi\left(\tau, p_{T}^{m i s s}\right)\right) & \text { VI. } & M\left(\tau, b_{H^{+}}\right) \\
\text {III. } & 1-\cos \left(\Delta \phi\left(H^{+}, b_{H^{+}}\right)\right) & \text {VII. } & \Delta R\left(\tau, b_{H^{+}}\right) \\
\text {IV. } & M\left(\tau, b_{H^{+}}\right) \cdot \Delta R\left(\tau, b_{H^{+}}\right) & &
\end{array}
$$

Table 2: Variables used for the Likelihood Discriminant. $p_{T}^{l t}$ is the $p_{T}$ of the leading track in the $\tau$ jet, $\tau$ denotes the $\tau$ jet in the event, $b_{H^{+}}$is the $b$ jet coming from the same top quark as the presumed $H^{+}$, and $M\left(\tau, b_{H^{+}}\right)$is the invariant mass of the $\tau$ jet and the $b_{H^{+}}$jet. $\Delta R=\sqrt{(\Delta \phi)^{2}+(\Delta \eta)^{2}}$ represents a distance.

Transverse mass calculation Following the cut on the likelihood, the transverse mass of the $\mathrm{H}^{+}$ candidate is calculated. A shape-based profile likelihood method is then applied to the transverse mass histogram (shown in Figure 1)(b) for a $H^{+}$mass of $130 \mathrm{GeV}$ ), to extract the significance of the signal hypothesis ${ }^{1}$. A more traditional, event-counting approach can also be followed by placing a cut on the transverse mass of the charged Higgs candidate (at $50 \mathrm{GeV}$ for the $90 \mathrm{GeV}$ mass point, at $60 \mathrm{GeV}$ for the 110 and $120 \mathrm{GeV}$ mass points, at $65 \mathrm{GeV}$ for the $130 \mathrm{GeV}$ mass points and at 75 $\mathrm{GeV}$ for the $150 \mathrm{GeV}$ mass point).

\subsection{Systematics}

Both theoretical and experimental sources of systematic errors were considered for this study. The effect of each source was evaluated individually by varying its expected uncertainty on an event-by-event basis. The overall systematic uncertainty on the signal was thereby determined to be $24 \%$. The systematic uncertainty on the $t \bar{t}$ background was found to be even larger, effectively removing most of the discovery potential. Therefore a method for data-driven background estimation [6] was developed, reducing the expected systematic uncertainty on the background to about $10 \%$.

\footnotetext{
${ }^{1}$ For more details see [5]
} 


\section{Results}

Table 3 shows the selection cut flow for five signal mass points and all studied backgrounds. The expected cross section after the trigger and after the first and second set of cuts is presented, as well as the relative efficiency of each step with respect to the previous one. Table 7 shows the final expected cross section, after the likelihood cut, as well as the relative efficiency of that cut. Given is also the expected cross section and efficiency for cutting on the transverse mass of the $H^{+}$candidate. Since the likelihood depends on the mass point for which it is evaluated, the final background rejection will also depend on the evaluated mass point.

\begin{tabular}{|c|c|c|c|c|c|c|}
\hline \multicolumn{3}{|c|}{ Channel } & All events & Trigger & Cuts I & Cuts II \\
\hline \multirow[t]{2}{*}{$H^{+}$} & $90 \mathrm{GeV}$ & [fb] & 38388 & 3384 & 404 & 136 \\
\hline & & efficie & & 0.088 & 0.119 & 0.337 \\
\hline \multirow[t]{2}{*}{$H^{+}$} & $110 \mathrm{GeV}$ & {$[\mathrm{fb}]$} & 27147 & 2871 & 286 & 112 \\
\hline & & effici & & 0.106 & 0.100 & 0.391 \\
\hline \multirow[t]{2}{*}{$H^{+}$} & $120 \mathrm{GeV}$ & {$[\mathrm{fb}]$} & 21363 & 2563 & 255 & 94 \\
\hline & & effici & & 0.120 & 0.099 & 0.368 \\
\hline \multirow[t]{2}{*}{$H^{+}$} & $130 \mathrm{GeV}$ & [fb] & 15666 & 2136 & 217 & 79 \\
\hline & & effic & & 0.136 & 0.102 & 0.364 \\
\hline \multirow[t]{2}{*}{$H^{+}$} & $150 \mathrm{GeV}$ & {$[\mathrm{fb}]$} & 5869 & 982 & 88 & 30 \\
\hline & & effic & & 0.167 & 0.089 & 0.342 \\
\hline \multirow{2}{*}{\multicolumn{2}{|c|}{$t \bar{t} \geq 1$ lepton }} & [fb] & $4.52 \cdot 10^{5}$ & 56287 & 852 & 307 \\
\hline & & effici & & 0.125 & 0.015 & 0.360 \\
\hline \multirow{2}{*}{\multicolumn{2}{|c|}{$t \bar{t}$ hadronic }} & [fb] & $3.81 \cdot 10^{5}$ & 1746 & 37 & 21 \\
\hline & & efficie & & 0.005 & 0.021 & 0.571 \\
\hline \multirow{2}{*}{\multicolumn{2}{|c|}{ single top }} & {$[\mathrm{fb}]$} & 112500 & 7700 & 63 & 17 \\
\hline & & efficier & & 0.068 & 0.008 & 0.277 \\
\hline \multirow{2}{*}{\multicolumn{2}{|c|}{$\mathrm{W}+$ jets }} & [fb] & 277800 & 15489 & 73 & 30 \\
\hline & & efficiency & & 0.056 & 0.005 & 0.409 \\
\hline \multirow{2}{*}{\multicolumn{2}{|c|}{ QCD dijets }} & {$[\mathrm{fb}]$} & $3.2 \cdot 10^{8}$ & $3.18 \cdot 10^{5}$ & 5 & 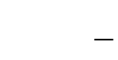 \\
\hline & & efficiency & & 0.001 & $1.7 \cdot 10^{-4}$ & \\
\hline
\end{tabular}

Table 3: Selection cut flow. For each sample, the cross sections after cuts are given in $\mathrm{fb}$ and for $\tan \beta=20$ in the first line and the relative cut efficiencies in the second line (in italics). Standard Model cross sections are given for all the backgrounds. There are insufficient Monte Carlo statistics for the QCD events - see discussion in the text for a cross section estimate. The final results, following the likelihood cut, are presented in Table 4 .

The discovery and exclusion contours obtained from applying the shape-based profile likelihood on the transverse mass histogram are shown in Figure 2. As can be seen, the discovery contour in the $m_{h}$-max scenario reaches down to below 20 in $\tan \beta$ and up to $H^{+}$masses of about $150 \mathrm{GeV}$, while the exclusion covers almost the entire range except for high masses (above 150 $\mathrm{GeV}$ ) and intermediate $\tan \beta$ (around 7). 


\begin{tabular}{ll|l||r|r|r|r}
\multicolumn{2}{c|}{ Channel } & \multicolumn{1}{l||}{ Cut } & \multicolumn{2}{c|}{ Signal } & \multicolumn{2}{c}{$t \bar{t} \geq 1 \quad e / \mu / \tau$} \\
& & & {$[\mathrm{fb}]$} & efficiency & {$[\mathrm{fb}]$} & efficiency \\
\hline$H^{+}$ & $90 \mathrm{GeV}$ & $\mathrm{LH}>0.6$ & 56.2 & 0.413 & 55.8 & 0.182 \\
& & $m T>50 \mathrm{GeV}$ & 35.3 & 0.628 & 32.1 & 0.574 \\
$H^{+}$ & $110 \mathrm{GeV}$ & $\mathrm{LH}>0.6$ & 53.6 & 0.478 & 52.7 & 0.172 \\
& & $m T>60 \mathrm{GeV}$ & 35.1 & 0.655 & 27.9 & 0.529 \\
$H^{+}$ & $120 \mathrm{GeV}$ & $\mathrm{LH}>0.6$ & 42.6 & 0.455 & 45.5 & 0.148 \\
& & $m T>60 \mathrm{GeV}$ & 32.5 & 0.764 & 29.0 & 0.636 \\
$H^{+}$ & $130 \mathrm{GeV}$ & $\mathrm{LH}>0.6$ & 38.3 & 0.483 & 50.7 & 0.165 \\
& & $m T>65 \mathrm{GeV}$ & 31.4 & 0.819 & 25.9 & 0.510 \\
$H^{+}$ & $150 \mathrm{GeV}$ & $\mathrm{LH}>0.8$ & 14.0 & 0.467 & 26.9 & 0.088 \\
& & $m T>75 \mathrm{GeV}$ & 9.3 & 0.662 & 10.3 & 0.385
\end{tabular}

Table 4: Final event selection results. Given are the cross sections after all cuts in $\mathrm{fb}$ and for $\tan \beta=$ 20 as well as the relative cut efficiencies. Standard Model cross sections are given for the backgrounds. Backgrounds not tabulated have been found to be negligible
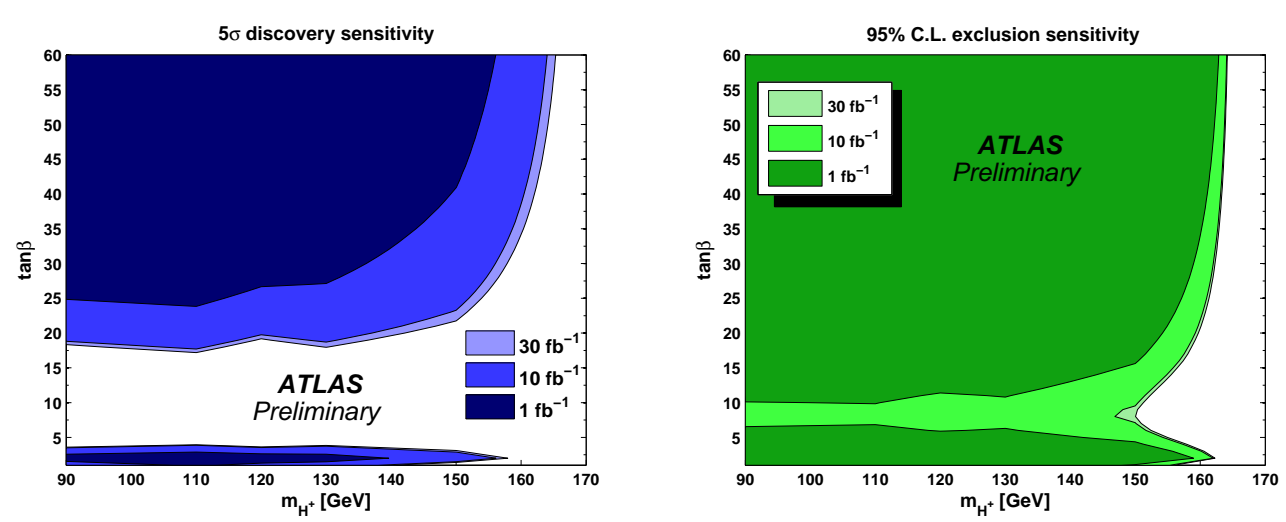

Figure 2: Discovery (left) and exclusion contour (right) for the $m_{h}$-max scenario. A systematic uncertainty of $10 \%$ for the background, and $24 \%$ for the signal has been included, along with the statistical uncertainties. The lines indicate a $5 \sigma$ significance for the discovery and a $95 \%$ CL for the exclusion contour. All significances have been obtained using the profile likelihood method.

\subsection{An estimate of the QCD background contribution}

QCD backgrounds are very important for this channel, as it is completely hadronic. However, the cross section for QCD dijet production at the LHC is enormous, making it impossible to simulate sufficient Monte Carlo events to be able to draw safe conclusions about the rejection of QCD backgrounds. As can be seen in Table 3, after the second set of cuts no MC QCD events remain (and as a matter of fact already following the first few cuts on the number of $\tau$ and $b$ jets, there are less than $10 \mathrm{MC}$ QCD events). Clearly this does not imply that there will not be any QCD events remaining at this point, but rather that an estimate will be needed in order to check that QCD events will not dominate this channel. 
A conservative upper limit on the number of QCD events can be obtained by substituting the $t \bar{t}$ efficiency ${ }^{2}$ for the QCD efficiency, starting from the cut after which too few QCD events survive (cut on number of $b$ jets). It is justified to assume that the expected efficiency of the QCD events is limited from above by the efficiency for $t \bar{t}$ events, since the $t \bar{t}$ background is much more similar to the signal final state which all cuts try to identify. At the same time it is also a very conservative estimate, since the second set of cuts was specifically designed to select the $t \bar{t}$ final state and discriminate against QCD backgrounds. Nevertheless, under these assumptions only about $55 \mathrm{fb}$ of QCD background would remain after the cut on the likelihood (and $30 \mathrm{fb}$ after cutting on the $H^{+}$transverse mass). This very conservative upper limit is of the same order of magnitude as the expected $t \bar{t}$ background, indicating that the background from QCD events can indeed be expected to be much less than the dominating $t \bar{t}$ background.

\section{Conclusions}

The discovery potential of ATLAS for a light $\mathrm{H}^{+}$in the hadronic mode has been investigated with fully simulated signal and background. Good separation between signal and background is obtained using selection cuts and a likelihood, which, given the relatively high cross section, allows for a substantial region of the MSSM parameter space to be probed, thus significantly contributing to the overall ATLAS discovery potential for $\mathrm{H}^{+}$.

\section{References}

[1] ATLAS Collaboration, Expected Performance of the ATLAS Experiment, Detector, Trigger and Physics, CERN-OPEN-2008-020, Geneva, 2008, to appear.

[2] M. Flechl Charged Higgs Prospects with ATLAS, this volume.

[3] C. Potter, Trigger Strategies for the Charged Higgs Boson, this volume.

[4] D.P. Roy, Phys. Lett. B 459 (1999) 607 - 614.

[5] O. Vitells, Statistical Combination of charged Higgs channels in ATLAS, this volume.

[6] T. Vickey, Data-driven Methods for the Estimation of ttbar Backgrounds to charged Higgs searches, this volume.

\footnotetext{
${ }^{2} \mathrm{Up}$ to the cut on the likelihood the efficiency for the all-hadronic $t \bar{t}$ background was used, while the $t \bar{t}>=1$ lepton efficiency was used thereafter.
} 